\title{
Ad-Hoc Reviewers from 2018
}

(c) Springer Science+Business Media, LLC, part of Springer Nature 2019

The Journal of Nonverbal Behavior greatly appreciates the assistance of the Editorial Board as well as the following ad hoc manuscript reviewers:

\begin{tabular}{|c|c|c|}
\hline Susan Andrzejewski & Sarah D. Gunnery & Michael Philipp \\
\hline Andrey Anikin & Amie Hane & Ken Prkachin \\
\hline Jo-Anne Bachorowski & Shlomo Hareli & Guillermo Recio \\
\hline Pascal Belin & Roel Hermans & Michaela Rohr \\
\hline Elizabeth Brey & Nicole Hirschmann & Mollie Alyssa Ruben \\
\hline Erik P. Bucy & Susan Hughes & Linda Ruekert \\
\hline Christopher Carpenter & Sylwia Hyniewska & Krystyna Rymarczyk \\
\hline Tanya L. Chartrand & Rachel Jack & Wataru Sato \\
\hline Mingyuan Chu & Philippe Jacquart & Disa Sauter \\
\hline Kenneth Craig & Kerri L. Johnson & Juliana Schroeder \\
\hline William Crano & Lucy Johnston & Danielle Shore \\
\hline Carlos Crivelli & Kerry Kawakami & Gary Shteynberg \\
\hline Nele Dael & Mahshid Khademi & Allison Louise Skinner \\
\hline Beatrice de Gelder & Emmanuelle Klienlogel & Marie Smith \\
\hline Leah Dickens & Miriam Kunz & Leanne ten Brinke \\
\hline Corine Dijk & Jessica Lakin & Linda Tickle-Degnen \\
\hline James Driskell & Ioana Latu & Elena Tsankova \\
\hline Wolfgang Einhäuser & Nadine Lavan & Amrisha Vaish \\
\hline Sally Farley & Juan David Leongomez & Ilja van Beest \\
\hline Ineke Fengler & Timothy Levine & John van der Kamp \\
\hline Agneta Fischer & Paige Lloyd & Elke van der Meer \\
\hline Kory Floyd & Valerie Manusov & Ishabel M. Vicaria \\
\hline Marie Ford & Andy Martinez & Andreas Voss \\
\hline Michael Forster & Steven McCaffes & Christian Wallraven \\
\hline Alan Fridlund & Marc Mehu & Auriel Washburn \\
\hline Ken Fujiwara & Maarten Milders & Caroline Whiting \\
\hline Mattia Gallotti & Abigail Millings & Oliver Wilhelm \\
\hline Gordon Gallup & Marcello Mortillaro & Adrienne Wood \\
\hline Maria Gendron & Steffan Nestler & Clea Wright Whelan \\
\hline Howard Giles & Paula Niedenthal & Mircea Zloteanu \\
\hline Jin Xun Goh & Marc D. Pell & \\
\hline
\end{tabular}

Publisher's Note Springer Nature remains neutral with regard to jurisdictional claims in published maps and institutional affiliations. 